\title{
UNIVERSITYOF
}

FORWARD

THINKING

WESTMINSTER用

WestminsterResearch

http://www.westminster.ac.uk/westminsterresearch

Two-Path All-pass Based Half-Band Infinite Impulse Response

Decimation Filters and the Effects of Their Non-Linear Phase

Response on ECG Signal Acquisition

Eminaga, Y., Coskun, A. and Kale, I.

NOTICE: this is the authors' version of a work that was accepted for publication in Biomedical Signal Processing \& Control. Changes resulting from the publishing process, such as peer review, editing, corrections, structural formatting, and other quality control mechanisms may not be reflected in this document. Changes may have been made to this work since it was submitted for publication. A definitive version was subsequently published in Biomedical Signal Processing \& Control, 31, 529-538.

Biomedical Signal Processing \& Control is available online at:

https://dx.doi.org/10.1016/j.bspc.2016.08.019

(C) 2016. This manuscript version is made available under the CC-BY-NC-ND 4.0 license http://creativecommons.org/licenses/by-nc-nd/4.0/

The WestminsterResearch online digital archive at the University of Westminster aims to make the research output of the University available to a wider audience. Copyright and Moral Rights remain with the authors and/or copyright owners.

Whilst further distribution of specific materials from within this archive is forbidden, you may freely distribute the URL of WestminsterResearch: ((http://westminsterresearch.wmin.ac.uk/).

In case of abuse or copyright appearing without permission e-mail repository@westminster.ac.uk 


\section{Two-Path All-pass Based Half-Band Infinite Impulse Response Decimation Filters and the Effects of Their Non-Linear Phase Response on ECG Signal Acquisition}

Yaprak Eminaga, Adem Coskun and Izzet Kale,

Department of Engineering, Applied DSP and VLSI Research Group, Faculty of Science and Technology

University of Westminster, 115 New Cavendish Street, London W1W 6UW

ENGLAND, UK.

y.eminaga@my.westminster.ac.uk, a.coskun@westminster.ac.uk,

kalei@westminster.ac.uk 


\begin{abstract}
This paper is based on the novel use of a very high fidelity decimation filter chain for Electrocardiogram (ECG) signal acquisition and data conversion. The multiplier-free and multi-stage structure of the proposed filters lower the power dissipation while minimizing the circuit area which are crucial design constraints to the wireless noninvasive wearable health monitoring products due to the scarce operational resources in their electronic implementation. The decimation ratio of the presented filter is 128 , working in tandem with a 1-bit $3^{\text {rd }}$ order Sigma Delta $(\Sigma \Delta)$ modulator which achieves $0.04 \mathrm{~dB}$ passband ripples and $-74 \mathrm{~dB}$ stopband attenuation. The work reported here investigates the non-linear phase effects of the proposed decimation filters on the ECG signal by carrying out a comparative study after phase correction. It concludes that the enhanced phase linearity is not crucial for ECG acquisition and data conversion applications since the signal distortion of the acquired signal, due to phase non-linearity, is insignificant for both original and phase compensated filters. To the best of the authors' knowledge, being free of signal distortion is essential as this might lead to misdiagnosis as stated in the state of the art. This article demonstrates that with their minimal power consumption and minimal signal distortion features, the proposed decimation filters can effectively be employed in biosignal data processing units.
\end{abstract} Keywords -- decimation filter; halfband allpass filter; multiplier free; non-linear phase; ECG; wearable health monitoring. 


\section{Introduction}

The ECG is a fundamental component in patient monitoring and diagnosis. Accurate ECG signal acquisition and its precise analysis are of great importance and have been subject to numerous research work [1]. ECG signals reflect transmembrane potential differences in myocardial cells, as a result of the ionic movement in and out of the extracellular space and can be measured between any two points on the body surface using electrodes. A standard clinical ECG signal is composed of waves at different frequency bands, each reflecting the electrical activation of different parts of the heart. These waves are known as the $\mathrm{P}, \mathrm{QRS}$, and $\mathrm{T}$ waves, representing the atrial depolarization, ventricular depolarization and repolarization respectively as shown in Fig.1. They have extremely low amplitude ranging from $100 \mu \mathrm{V}$ to $5 \mathrm{mV}$ and low diagnostic frequency bandwidth between 0.05 to $100 \mathrm{~Hz}$ [2]. Diagnosis of the cardiac health conditions mostly rely on the assessment of the ECG data based on the interbeat timing and wave amplitudes. Different types of arrhythmia can be distinguished by the morphological and beat-to-beat interval variations and/or missing beats. The standard clinical features of ECG waves for a healthy adult male in sinus rhythm are presented in Table I [2]. These metrics depend on several factors such as age, gender, heart rate, respiration patterns and diseases. [2]. 


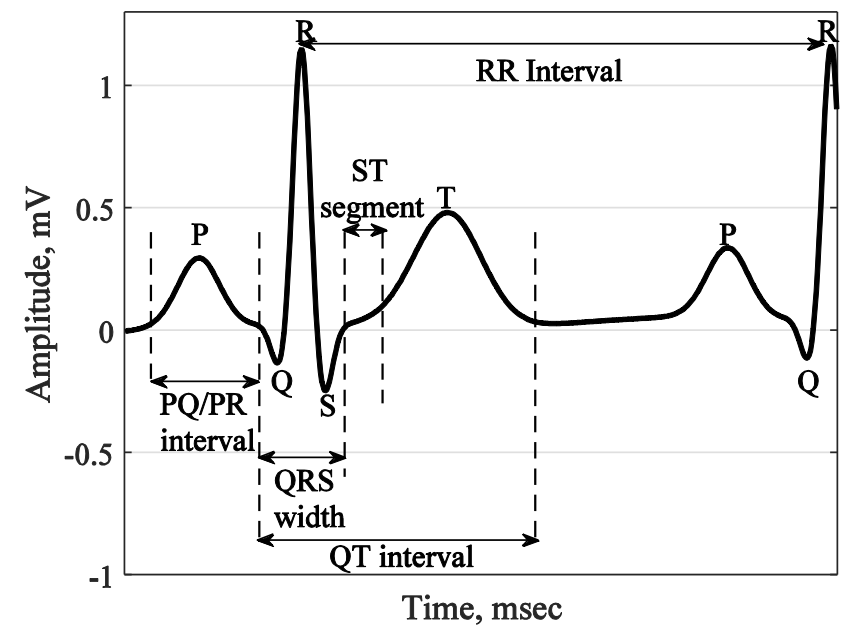

Fig.1. Time domain features of an ECG signal - P, QRS, and T respresent the atrial depolarization, the ventricular depolarization and atrial and ventricular respolarization respectively [3].

TABle I. NORMAL VAlUES OF TyPICAL LEAD II ECG FEATURES OF A HEALthy MALE SUBJECT IN SinUs RHYTHM AT 60 BPM [2]

\begin{tabular}{|ccc|}
\hline Feature & Normal Value & Normal Limit \\
P width & $110 \mathrm{~ms}$ & $\pm 20 \mathrm{~ms}$ \\
PQ/PR interval & $160 \mathrm{~ms}$ & $\pm 40 \mathrm{~ms}$ \\
QRS width & $100 \mathrm{~ms}$ & $\pm 20 \mathrm{~ms}$ \\
QT interval & $400 \mathrm{~ms}$ & $\pm 40 \mathrm{~ms}$ \\
ST segment & $70 \mathrm{~ms}$ & $\pm 10 \mathrm{~ms}$ \\
P amplitude & $0.15 \mathrm{mV}$ & $\pm 0.05 \mathrm{mV}$ \\
QRS height & $1.5 \mathrm{mV}$ & $\pm 0.5 \mathrm{mV}$ \\
ST level & $0 \mathrm{mV}$ & $\pm 0.1 \mathrm{mV}$ \\
T amplitude & $0.3 \mathrm{mV}$ & $\pm 0.2 \mathrm{mV}$ \\
\hline
\end{tabular}

Due to low amplitude characteristics, 10 to 16 bits of resolution is required in order to represent the acquired ECG data accurately in the digital domain. Thus, the Sigma-Delta $(\Sigma \Delta)$ oversampled Analog-to-Digital Converters (ADCs) are well suited for the digitization process, since they provide high resolution and dynamic range for lowbandwidth biomedical signals with simple hardware architectures [4]. The $\Sigma \Delta$ ADCs incorporate two sections which are the modulator and digital filter. The $\Sigma \Delta$ modulator reduces the quantization noise in the signal band by moving it to higher frequencies by means of oversampling and noise shaping. Digital processing of the raw high-rate bit 
stream from the modulator is computationally complex, resulting in high power consumption and expensive digital circuitry. Therefore, a decimation filter is used to reduce the sampling rate while preserving the resolution by filtering the high frequency noise and downsampling. The wearable medical embedded systems face many design challenges, most important ones being the operating lifetime, area occupied, power consumption and affordability [5]. This work proposed a decimation filter chain for ECG signal acquisition which provides a highly efficient filtering performance by introducing minimal signal distortion, contributing towards overcoming the aforementioned challenges. The proposed design employs cascaded Slink filter (generally spuriously referred as CIC filter in the state of the art $[6,7]$ ), two path allpass (AP) based half-band (HB) Infinite Impulse Response (IIR) filters and a Slink rolloff compensator [8-14].

Several decimation filters are proposed for biosignal acquisition systems. Some employed multi-stage FIR decimation filters with very high filter orders, and therefore necessitating extra processing in terms of arithmetic operations [15-19]. Some used single stage decimation filter which is solely a Slink filter [20].However, using a single Slink filter with high decimation ratio comes at a high cost, does not easily provide the required stopband attenuation for supressing the high frequency noise, and exhibits a high roll-off in the passband region which degrades the in-band signal features. In other studies, four stage decimation filters that have high power consumption and hardware complexity are used $[21,22]$. In $[23,24]$ single stage FIR decimation filters are used, in which the filter coefficients are optimized in order to match the analog loop filter response of the modulator. Single stage decimation with high decimation rate requires sharper transition, high stopband attenuation and thus higher filter order. Whereas, the 
Canonical Signed Digit (CSD) representation of filter coefficients is another alternative for reducing the complexity of the decimation filters. This representation uses series of subtractions and additions in order to accomplish the multiply operation. Although the number of multiplier units are decreased by this method, the need for high order digital filters in the decimation stage is not avoided $[1,25]$. The aforementioned filters are all designed by using high order FIR filters which inevitably increased the power consumption and hardware complexity. In addition, in $[1,26]$ three stage decimation chains each employing a slink, an FIR and an IIR filter are proposed. However, due to the non-linear phase of the IIR filter, an additional phase equalizer is applied following the decimation chain. Thus, even though the overall filter order is reduced, compared to FIR filters, the hardware complexity and power consumption is increased by the use of the equalizer.

This paper describes the design of a two-path AP based HB IIR filters within a decimation chain which are deployed in various applications in which they required small number of filter coefficients and thus avoiding higher orders [7-13]. IIR filters are renowned for their computational efficiency however at the expense of having a nonlinear phase response which may result in waveform distortions in time-domain. The work reported here studies the non-linear phase effects of these filters on ECG data. For further investigation phase compensation filters are implemented using low-complexity cascaded AP filter sections and results from the original and phase corrected filters are comparatively studied. To the best knowledge of the authors this is a first in the open literature.

The rest of the paper introduces the detailed structure of the proposed decimation filter along with the magnitude, phase and group delay characteristics. The following section 
further provides information on phase compensation filter design and introduces the databases used for simulation, test and evaluation purposes. The paper concludes with the results and discussion sections.

\section{Methodology and Experimental Evaluation Setup}

In this section the proposed decimation filter structure along with filters' group delay response are first introduced. Following these, the design of phase compensation filters and the methods used for evaluating and testing the performance of the proposed design on ECG data are described.

\subsection{The Proposed Decimation Filter Structure}

The proposed decimator (filter followed by downsampling) is designed to achieve a decimation ratio of 128 in order to demodulate the 1-bit output stream from a third$\operatorname{order} \Sigma \Delta$ modulator. It is composed of four cascaded multiplier free stages, which are a fourth-order Slink filter, two fifth-order two-path AP based HB IIR filters and a first order Slink compensation filter. The overall decimator is designed to achieve $0.04 \mathrm{~dB}$ passband ripples and $-74 \mathrm{~dB}$ stopband attenuation. The behavioural structure of the cascaded decimation filter incorporating the decimation ratios at each stage is shown in Fig.2.

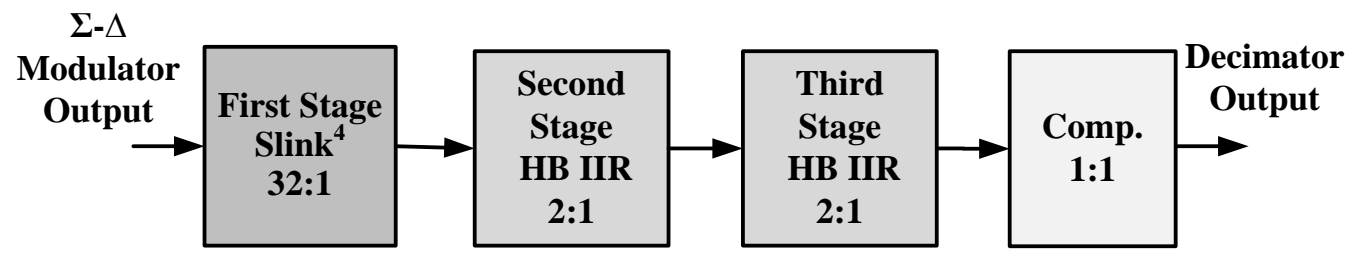

Fig. 2. Behavioural structure of the decimation filter, incorporating the $4^{\text {th }}$ order Slink, two 2-path AP based HB IIR, and Slink compensation filters. 


\subsubsection{The Slink Filter}

A fourth order Slink filter with a decimation ratio of 32 is the first stage of the proposed design that has linear phase with a multiplier free structure and the z-domain transfer function is given in (1) [6].

$$
H_{\text {slink }}(z)=\frac{1}{32^{4}}\left(\frac{1-z^{-32}}{1-z^{-1}}\right)^{4}
$$

The gain of the filter is required to roll-off at a faster rate than the $\Sigma \Delta$ modulation noise rises, and therefore the order of the filter has to be one higher than the $\Sigma \Delta$ modulator's order. On the contrary, a filter with higher order causes higher roll-off in the band of interest and a lower order filter will not provide the required stopband characteristics resulting in aliasing in the signal band [27]. The Slink filter incorporates two sections with the opportunity of shifting the downsampler in between these two sections. The two sections are 4 cascaded accumulators and 4 cascaded differencers. The shifted downsampler enables the differencers to operate at 32 times slower rate than the input, reducing the power consumption, which makes it well suited for applications requiring high decimation factors and low circuit complexity and power consumption [7]

\subsubsection{Two-Path All-pass based Half-Band Infinite Impulse Response Filters}

In order to provide computationally efficient second and third stages of the decimation chain shown in Fig. 2, very high fidelity minimum phase two-path AP based HB IIR filters are designed where at each stage the input signal is decimated by two. The filters described here are formed by two-parallel paths composed of second order AP filters with multiplier free structures. The delayer in the bottom path of the recursive polyphase structure creates an increasing phase difference between the two paths. This difference reaches $90^{\circ}$ at $v_{c}$ and $180^{\circ}$ at $v_{s}$ which define the cut-off and the stopband 
frequencies respectively. Since $A_{i}(z)$, given in (2), is rational and sparse, the downsamplers can be shifted before the AP sections by using the Noble identity in order to avoid unnecessary sample operations [28] as shown in Fig. 3. The coefficients of the AP sections in the second and the third decimation stages are designed to be powers of two $\left(\alpha_{1}=0.125\right.$ and $\left.\alpha_{2}=0.5625\right)$. These coefficients eliminate the need for multipliers by replacing them with shift and add operations, as shown in Fig. 4 (a) and (b), where " >>" represents a binary shift operation along with a number indicating the number of shifts. The z-domain transfer function for both AP sections is $[9,29]$,

$$
A_{i}(z)=\frac{\alpha_{i}+z^{-2}}{1+\alpha_{i} z^{-2}}
$$

where $i=1,2$ and $\alpha_{i}$ represents the coefficients of the AP filters. Each of the proposed HB IIR filters achieves $0.47 \mu \mathrm{dB}$ passband ripples in the region of signal activity and $70 \mathrm{~dB}$ stopband attenuation with minimal hardware complexity.

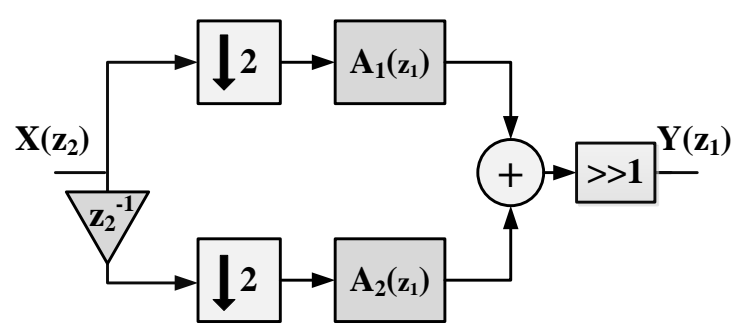

Fig. 3. Two-path AP based HB IIR Decimator structure, incorporating AP filters with zdomain transfer functions $\mathrm{A}_{1}\left(\mathrm{z}_{1}\right)$ and $\mathrm{A}_{2}\left(\mathrm{z}_{1}\right)$ in the top and bottom paths respectively. $z_{2}$ and $z_{1}$ represents the fast and slow rate delayers respectively. 


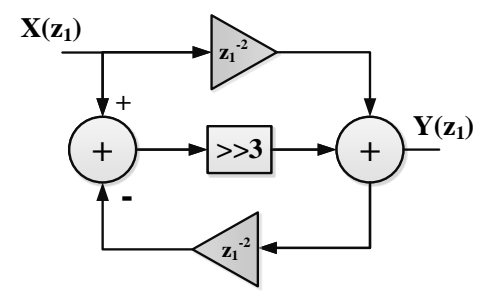

(a)

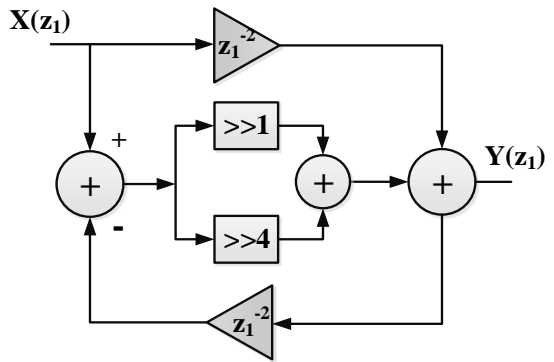

(b)

Fig. 4. First order AP filter structures for (a) $\mathrm{A}_{1}\left(\mathrm{z}_{1}\right)$ in the top branch with $\alpha_{1}=0.125$ and (b) $\mathrm{A}_{2}\left(\mathrm{z}_{1}\right)$ in the bottom branch with $\alpha_{2}=0.5625$.

\subsubsection{The Slink Roll-off Compensation Filter}

The Slink compensator is the last stage in the proposed design, exhibiting a passband response that is the inverse of the Slink filter in order to compensate the amplitude rolloff in the band of interest (dc to half Nyquist) caused by the Slink filter. The compensator has a similar structure with the AP sections given in Fig. 4 whereas only one delayer is required as shown in Fig. 5 [6, 31]. This filter also has a multiplier free structure, since the only coefficient is designed as power of two and can be implemented as a shift operation.

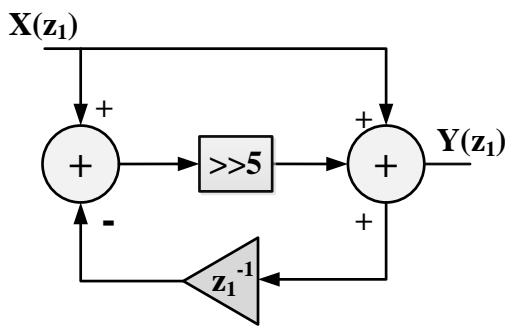

Fig. 5. Slink roll-off compensation filter structure with coefficient $\alpha_{c}=0.03125$.

\subsubsection{Decimation Chain Magnitude Response}

The decimation filters introduced in the previous sections were implemented using MATLAB in order to validate the overall system performance. The overall decimation chain achieves $0.04 \mathrm{~dB}$ passband ripples and $-74 \mathrm{~dB}$ stopband attenuation. Fig. 6. (a) 
and (b) present the fullband magnitude response of the decimation chain at oversampled and output data rate. Fig. 6. (b) further presents the Slink (blue) and Slink compensation filters'(red) magnitude characteristics in the band of interest.

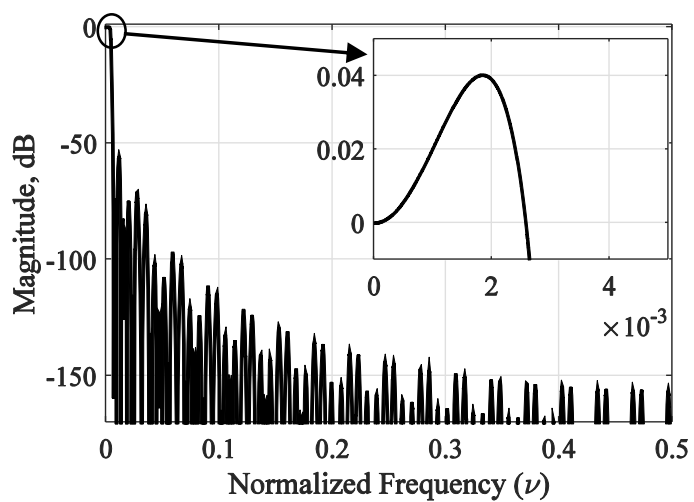

(a)

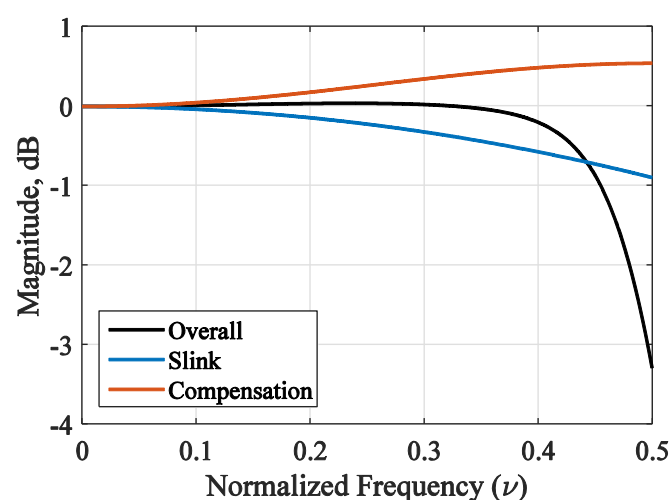

(b)

Fig. 6. Full band magnitude response of the overall decimation filter, (a) at input rate (zoomed into the passband region) and (b) at the output rate (decimated) along with the Slink (blue) and Slink compensation (red) filters' magnitude response.

\subsubsection{Phase Characteristics of Two-Path All-pass Based Half-band Polyphase IIR Filter}

IIR filters are well known for their non-linear phase response which should be compensated in order to avoid any phase distortion on the signal to be processed. The filter group delay is a measure of the linearity of the filter's phase response which is defined as the negative derivative of the phase of the system with respect to frequency i.e. $\tau(v)=-\frac{1}{2 \pi} \frac{d \phi(v)}{d v}$ where $\phi(v)$ is the filter phase and $v=f / f s$ is normalized frequency, $f s$ being the sampling frequency [30].

The group delay contribution of the second stage and third stage filters (Fig. 2), both of which are chosen to have the same structural and coefficient content as depicted in Fig.3 and Fig.4, to the overall group delay is

$$
\tau_{I I R}(v)=\underbrace{N_{1} \tau_{H B}\left(N_{1} v\right)}_{2^{n d} \text { stage HB IIR }}+\underbrace{2 N_{1} \tau_{H B}\left(2 N_{1} v\right)}_{3^{\text {rd }} \text { stage HB IIR }}
$$




$$
\tau_{H B}(v)=\frac{1}{2}+2 \sum_{k=1}^{K} \tau_{k}(v)+\Delta
$$

where $N_{l}$ is the downsampling ratio at the first stage of the decimation filter and $K$ is the number of paths in the filter structure. In (4), $\tau_{k}(v)$ represents the group delay contribution of the $k^{\text {th }}$ AP filter with coefficient $\alpha_{k}$ to the overall group delay of the AP based HB IIR decimation filter $\tau_{H B}(v)$, which can be formulated as follows

$$
\tau_{k}(v)=\frac{1-\alpha_{k}^{2}}{1+\alpha_{k}^{2}+2 \alpha_{k} \cos (4 \pi v)}
$$

and $\Delta=\sum \delta\left(v_{z}\right)$ is the summation of the delta functions in the group delay occurring at frequencies $v_{z}>0.25$ corresponding to the filter zeros [30]. Since the frequency components beyond the cut-off frequency correspond to filter's stopband region, the parameter $\Delta$ in (4) can be ignored. The total group delay for the aforementioned filters results in a bell-like shape that can be formulated as $\tau_{H B}(v)=\frac{1}{2}+\tau_{1}(v)+\tau_{2}(v)$ according to (4), for $K=2$ and ignoring the delta functions at the filter's stopband. Here $\tau_{l}(v)$ and $\tau_{2}(v)$ are the group delay functions of the AP filters in the top and bottom branches. The group delay of the two-path AP based HB IIR filter with non-linear phase response is presented in Fig. 7. 


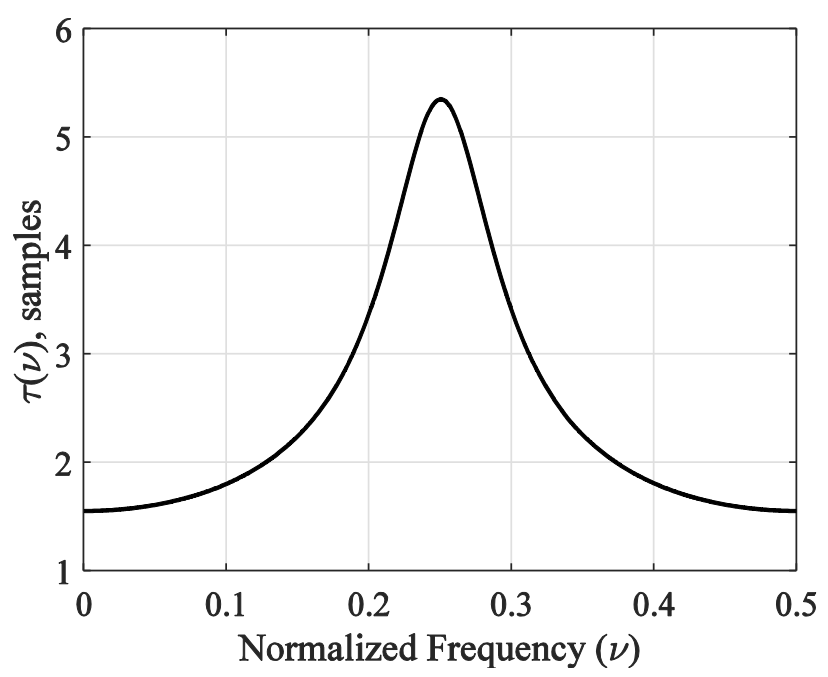

Fig. 7. The group delay of the two-path AP based HB IIR filter with the AP section coefficients $\alpha_{1}=0.125$ and $\alpha_{2}=0.5625$.

\subsection{Phase Compensation}

Linear phase is desired for most of the systems especially the biomedical applications since the temporal characteristics of a biosignal is of great importance due to diagnostic purposes. Non-linear phase results in a non-constant group delay which introduces different amount of time delays at different frequencies which may result in a distortion in the time domain amplitude of a signal.

Phase non-linearity can be compensated by designing cascaded AP correctors with a phase response which is approximately opposite of that for the polyphase filter [30]. The aim is to achieve an almost constant group delay response in the band of interest. A general transfer function for a $\mathrm{K}$ section compensator is

$$
H_{c}(v)=\frac{\alpha_{c 0}+z^{-2}}{1+\alpha_{c 0} z^{-2}} \prod_{m=0}^{K-2} \frac{\alpha_{c m}+z^{-\frac{2^{m-1}}{v_{c}}}}{1+\alpha_{c m} z^{-\frac{2^{m-1}}{v_{c}}}}
$$

where $\alpha_{c 0}$ and $\alpha_{c m}$ are the coefficients of a single second order and higher order correctors respectively. In order to study the non-linear phase effects of the proposed 
AP based HB IIR filters, a second order single corrector and a four section compensator are designed according to the detailed algorithm provided in [30]. Due to high sampling rate at the second stage of decimation, the phase non-linearity in the band of interest (i.e. the group delay peak-to-peak difference) is small however, it grows quickly as the sampling rate reduces at the third stage. Thus, a single AP corrector at the second stage is sufficient whereas, a 4 section corrector is required for the third stage in order to achieve a better correction in the band of interest. In this study, the phase is compensated up-to $v_{\mathrm{c}}=0.125$ which reduces the peak-to-peak difference in the band of interest (in this case for $v_{\mathrm{c}}=0.125$ ) from 0.431 to 0.0041 . In other words peak-to-peak error is reduced 106.3 times, calculated by (7) [30].

$$
E=\frac{\tau_{b}(v)_{\max }-\tau_{b}(v)_{\min }}{\tau_{a}(v)_{\max }-\tau_{a}(v)_{\min }}, \quad \text { where } 0 \leq v<v_{\mathrm{c}}
$$

where $\tau_{b}(v)$ and $\tau_{a}(v)$ are the group delays before and after phase correction respectively.

Fig. 8 (a) presents the normalized group delay responses of the original filter (blue) versus the phase corrected filter (black) via a single section AP corrector (red). Similarly, Fig. 8 (b) illustrates the group delay responses of the original (blue) and corrected filter (black) with 4 section AP corrector (red). 


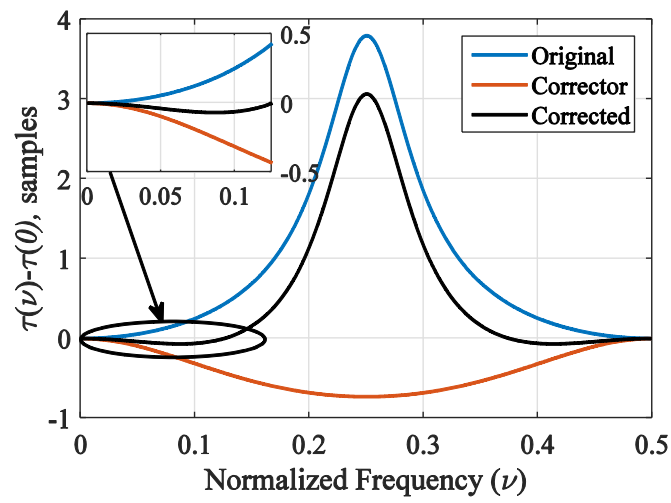

(a)

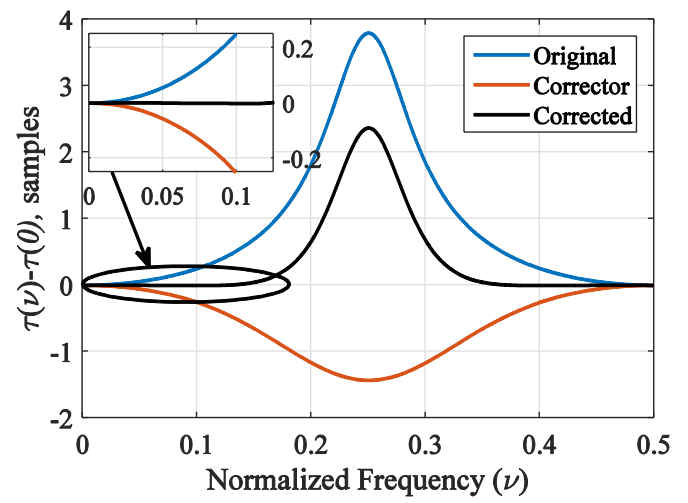

(b)

Fig. 8 The two-path polyphase IIR filter's normalized group delay response corrected using (a) a single section corrector and (b) a 4 section corrector along with the original filter's (blue), and the phase compensator's (red) group delay responses.

\subsection{T-Wave Alternans Challenge Database and MIT-BIH Arrhythmia}

\section{Database}

For evaluating the performance of the proposed decimation filter with ECG data, 10 second long recordings from two databases were used. The T-Wave Alternans Challenge (TWAC) Database provides ECG recordings at sinus rhythm which were acquired and digitized at $500 \mathrm{~Hz}$ by a typical 12-lead standard ECG from healthy subjects [32]. In addition, the MIT-BIH database provides ECG data with a wide range of arrhythmia and beat morphology variations, which were recorded via a two-channel recorder and digitized at $360 \mathrm{~Hz}[33,34]$.

Spectral analysis was carried out for both datasets by taking the averages of the power spectral densities (PSDs) of each data set for each Lead in order to determine the frequency content distribution of the data records. Results from the TWAC Database records showed that more than $99 \%$ of the average power is concentrated in the frequency band of $0-50 \mathrm{~Hz}(v=0-0.1)$ Fig. 9 (a) presents the average power of 12 Leads (named through I to V6) at the corresponding frequency band for record twa55. Fig.9 (b) shows the average power in several frequency bands of Lead II records of 13 
different heart conditions, obtained from MIT-BIH Arrhythmia database along. These heart conditions are chosen according to their density of occurrence such as atrial fibrillation, ventricular fibrillation and tachycardia. In the presence of different arrhythmias and conduction abnormalities, similar to the previous observation, more than $99 \%$ of the average power is concentrated in the frequency band of $0-45 \mathrm{~Hz}(v=$ 0 - 0.125). The $60 \mathrm{~Hz}$ power-line interference (USA standard) and its second harmonic can be observed as peaks in the corresponding plots.

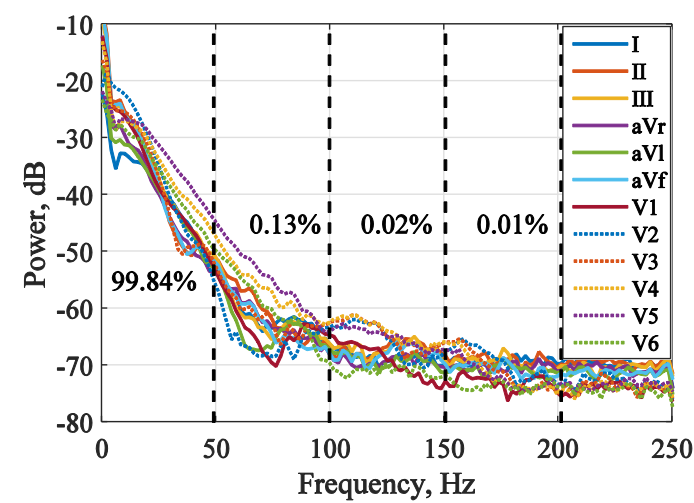

(a)

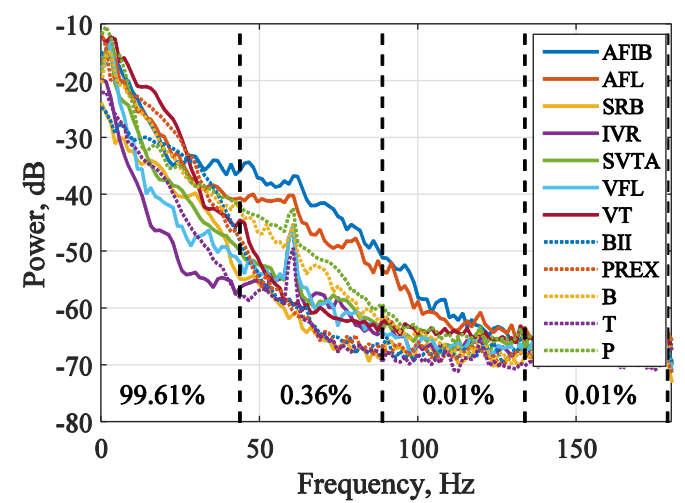

(b)

Fig.9. PSD of 10 seconds long (a) 12 lead recordings (from Lead I to V6) of record twa55 in sinus rhythm obtained from TWAC Database and (b) 13 Lead II recordings from MIT-BIH Arrhythmia Databse with various conduction abnormalities and beat morphologies. (AFIB :Atrial fibrillation, AFL: Atrial flutter, SBR: Sinus bradycardia, IVR: Idioventricular rhythm, SVTA: Supraventricular tachyarrhythmia, VFL: Ventricular flutter, VT: Ventricular tachycardia, BII: $2^{\circ}$ heart block, PREX: Pre-excitation, B: Ventricular bigeminy, T: Ventricular trigeminy and P: Paced rhythm)

In addition, the spectral resemblance between each of the leads and consecutive 10 second time segments of the same lead for both sets of data is studied by measuring the cross spectral coherence (CSC) which is defined as [2],

$$
C_{x y}=\frac{\left|P_{x y}\right|^{2}}{P_{x x} P_{y y}}
$$


where $P_{x x}$ and $P_{y y}$ are the power spectral estimates of $x$ and $y$ respectively, and $P_{x y}$ is the cross spectral estimate of $x$ and $y$. The coherence value shows the degree of spectral resemblance of $x$ to $y$ at each frequency, which ranges between 0 and 1 [2]. The CSC results are almost 1 for each lead and different time instances showing very high spectral resemblance among each other. Therefore, the performance of the decimation filters on one channel of a 10 second segment of ECG signal is a good indication for its performance on the overall system and longer recordings.

\subsection{Error Measures}

The performance of the proposed filter without and with phase correction can be quantified by measuring the filter input and output signal similarities in time domain (after time alignment) by calculating the cross correlation and Root Mean Square Error (RMSE) among each other which is defined in (9) and (10) [2, 35]. The correlation coefficient values are normalised between 0 and 1 which indicate no similarity and exact match respectively. The complement of the correlation coefficient is used as a measure for the waveform dissimilarities and is insensitive to variations in gain [36]. Thus, the RMSE is used for measuring the variations in the amplitude of the input $x[\mathrm{n}]$ and output $y[\mathrm{n}]$.

$$
R(x, y)=\frac{C_{x y}}{\sqrt{C_{x x} C_{y y}}}
$$

where, $C_{x y}$ is the covariance between $x[\mathrm{n}]$ and $y[\mathrm{n}], C_{x x}$ and $C_{y y}$ are the auto-covariance of $x[\mathrm{n}]$ and $y[\mathrm{n}]$ respectively.

$$
R M S E=\frac{1}{N} \sqrt{\sum_{n=1}^{N}(x[n]-y[n])^{2}}
$$


where, $N$ is the number of samples, $x[\mathrm{n}]$ is the ECG data records, and $y[\mathrm{n}]$ is the output from the decimation chain. The spectral similarities of the corresponding signals are quantified by calculating the distortion ratio (DR) and the CSC, given in (11) and (8) respectively. The CSC is a measure that is not effected by the signal morphology and amplitude, quantifying the steadiness of the input and output signal phases as a function of frequency [36].

$$
D R=\left[\frac{\sum(Y(f)-X(f))^{2}}{\sum X(f)^{2}}\right]^{1 / 2}
$$

where $X(f)$ and $Y(f)$ are the spectral magnitude of the input and output of the decimation chain.

\section{Experimental Results}

In order to test and evaluate the performance of the proposed decimation filter for ECG signal acquisition, ECG data, described in Section 2.3, were fed into a single-loop $3^{\text {rd }}$ order 1-bit $\Sigma \Delta$ modulator and modulated at a rate of 128 times faster than the Nyquist sampling frequency which is then filtered and decimated. The behavioural fixed-point models of the aforementioned filters were implemented through MATLAB.

The simulations were run for the 10 second long Lead II recordings of two different databases. First dataset includes 6 recordings (records twa39, twa46, twa55, twa60, twa90 and twa99) from healthy subjects in sinus rhythm and the second data set includes 13 each representing different arrhythmias and beat morphologies as depicted in Fig 9. Both datasets are decimated by the decimation filters described in Section 2.1 with and without phase compensation. 
The decimation filter performance is first evaluated by measuring the SNR at the output of the $\Sigma \Delta$ modulator and the decimation chain without and with compensation, under the sampling rates of $46.08 \mathrm{kHz}$ and $64 \mathrm{kHz}$. The results were obtained by feeding a 50 $\mathrm{Hz}$ sine wave with an added $60 \mathrm{~dB}$ white noise to the $\Sigma \Delta$ modulator and the signal bandwidths were chosen as $360 \mathrm{~Hz}$ and $500 \mathrm{~Hz}$ for the aforementioned sampling conditions respectively. The SNR (in the band of interest) at the output of the modulator and the decimation chains without and with phase compensation at the sampling rate of $64 \mathrm{kHz}$ were $80.2 \mathrm{~dB}, 80.1 \mathrm{~dB}$ and $80.1 \mathrm{~dB}$ respectively. The proposed decimation filter successfully preserved the SNR in the band of interest after the decimation process. In addition, SNRs obtained after decimating the modulated signal with the decimation filters without and with compensation demonstrated that the compensation filters do not contribute to the in band noise attenuation. Fig. 10 (a) and (b) present the PSDs and SNRs of the modulator and decimator outputs, respectively.

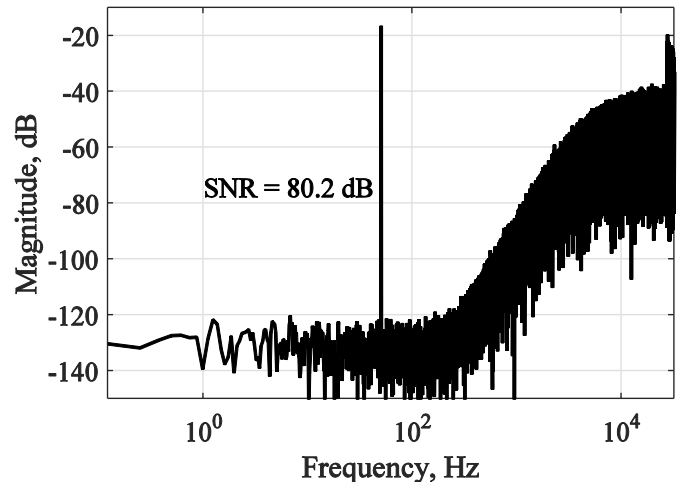

(a)

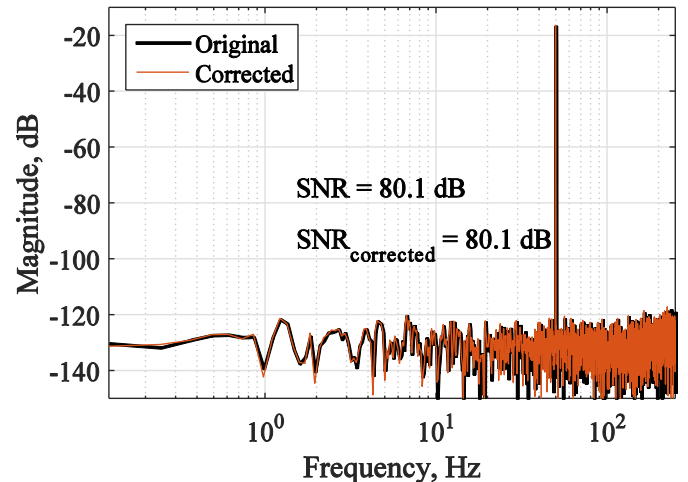

(b)

Fig. 10. Power spectrum measurments within the signal bandwidth of $500 \mathrm{~Hz}$ for sampling rate of $64 \mathrm{kHz}$, (a) $\Sigma \Delta$ modulator output and (b) decimation chain output (without phase compensation (black) and with phase compensation (red).

Normalized group delay introduced by the final three stages of the decimation chain (two AP based HB IIR filters and a slink compensator), the compensated group delay 
(up to $v=0.125$ ), filter magnitude response at the decimation chain output and the PSD of a Lead II ECG record (record twa55) from a healthy subject are shown in Fig. 11.

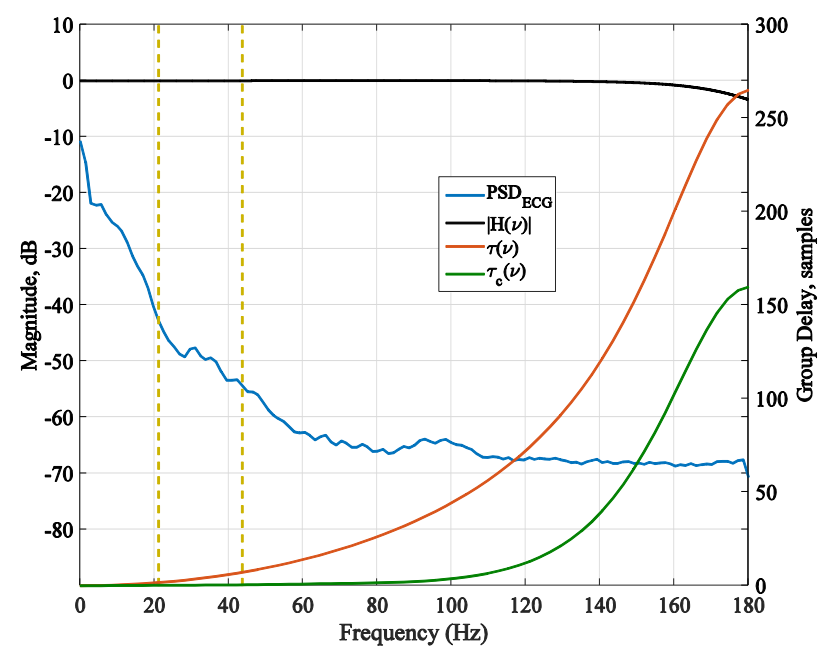

Fig. 11 PSD of Lead II recording of record twa55 at sinus rhythm (blue) versus the group delay variation with (green) and without phase compensation (red) filters, $f_{l}=22.5 \mathrm{~Hz}$ $\left(v_{1}=0.0625\right)$ and $f_{2}=45 \mathrm{~Hz}\left(v_{2}=0.125\right)$ indicated by the yellow lines at $f_{s_{2}}=46.08 \mathrm{kHz}$.

The variations in the total group delay of the filters are calculated in the specified frequency bands (yellow lines in Fig. 11). Table II provides the group delay variations in number of samples and in $\mu$ seconds at two different sampling rates $f_{s_{1}}=64 \mathrm{kHz}$ and $f_{s_{2}}=46.08 \mathrm{kHz}$ for TWAC Database and MIT-BIH Arrhythmia Database respectively.

TABLE II. VARIATION IN THE GROUP DELAY IN NORMALIZED FREQUENCY BANDS OF

$$
v_{B 1}=0-0.0625 \text { AND } v_{B 2}=0-0.125 \text {. }
$$

\begin{tabular}{|c|c|c|c|c|c|}
\hline \multirow{2}{*}{$\begin{array}{c}\text { Normalized } \\
\text { Frequency }\end{array}$} & \multicolumn{2}{|c|}{ Without compensation } & \multicolumn{3}{|c|}{ With Compensation } \\
\hline & Samples & $\mu \mathrm{Sec} \quad \mu \mathrm{Sec}$ & Samples & $\mu \mathrm{Sec}$ & $\mu \mathrm{Sec}$ \\
\hline Band $\left(v_{B}\right)$ & & $\left(\mathrm{fs}_{1} *\right)\left(\mathrm{fs}_{2} * *\right)$ & & $\left(\mathrm{fs}_{1}\right)$ & $\left(\mathrm{fs}_{2}\right)$ \\
\hline $0-0.0625$ & 1.86 & $40.28 \quad 29$ & 0.22 & 4.8 & 3.44 \\
\hline $0-0.125$ & 7.61 & 165.15119 & 0.49 & 10.63 & 7.66 \\
\hline
\end{tabular}


As mentioned in Section 2.3 and shown in Fig. 9, more than $99 \%$ of the power of the ECG data is concentrated in the frequency range of the $v=0-0.125$ for both datasets, thus group the delay variations are explored in this frequency range. According to the upper and lower limits of wave durations given in Table I, the group delay variation caused by the decimation filters are relatively low for the frequencies up to $v=0.125$. The maximum variation the signals are exposed to are $165 \mu \operatorname{secs}\left(f s_{2}=46.08 \mathrm{kHz}\right)$ and $119 \mu \operatorname{secs}\left(f_{s_{1}}=64 \mathrm{kHz}\right)$ without compensation, and $10.63 \mu \operatorname{secs}\left(f_{s_{2}}=46.08 \mathrm{kHz}\right)$ and $7.66 \mu \operatorname{secs}\left(f_{s_{1}}=64 \mathrm{kHz}\right)$ with compensation. These deviations are comparatively low regarding to the wave durations and normal limits for standard ECG features, thus the group delay variation due to phase non-linearity without compensation in this frequency range is negligible.

The first experimentation incorporated the data obtained from healthy subjects which were digitized at $500 \mathrm{~Hz}$ and oversampled by a factor of 128 (i.e. $64 \mathrm{kHz}$ ). Fig 12. (a) and (b) illustrate the input (record twa55) versus output from decimation chain without and with phase compensation respectively. As it can be observed from Fig. 12, there is no visible error between two filter outputs. This amplitude error can be visualized from Fig. 13 obtained by subtracting the original and phase compensated outputs from the original input. In Fig. 13, a relatively large overshoot with a decaying manner can be observed which is due to the transient response of the IIR filters. 


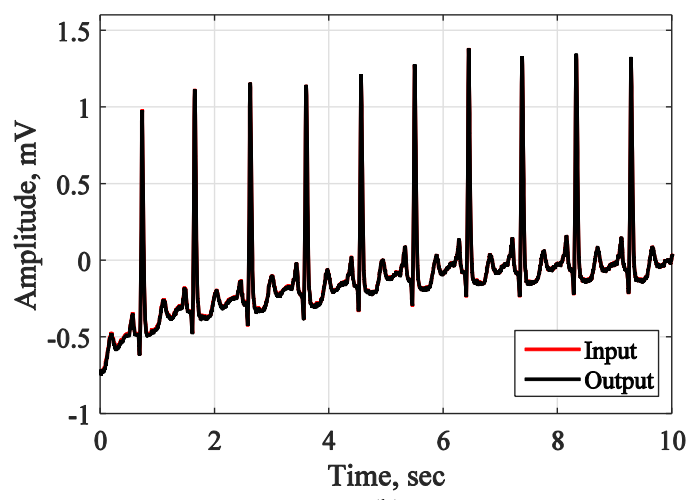

(b)

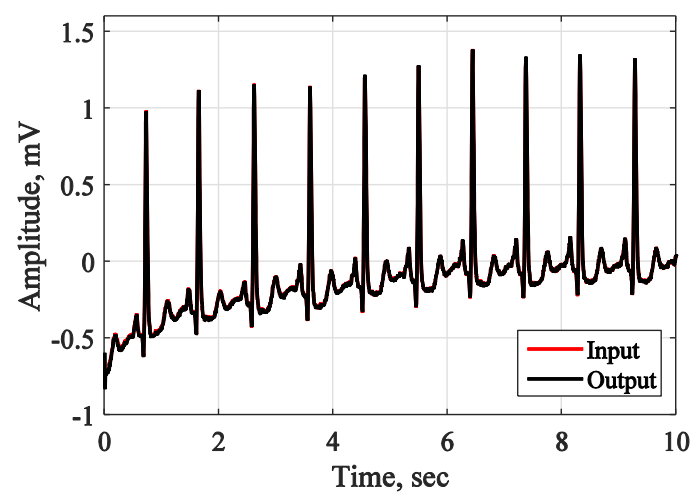

(a)

Fig. 12. Decimation chain output (black) versus the input(red) (a) without phase compensation and (b) with phase compensation.

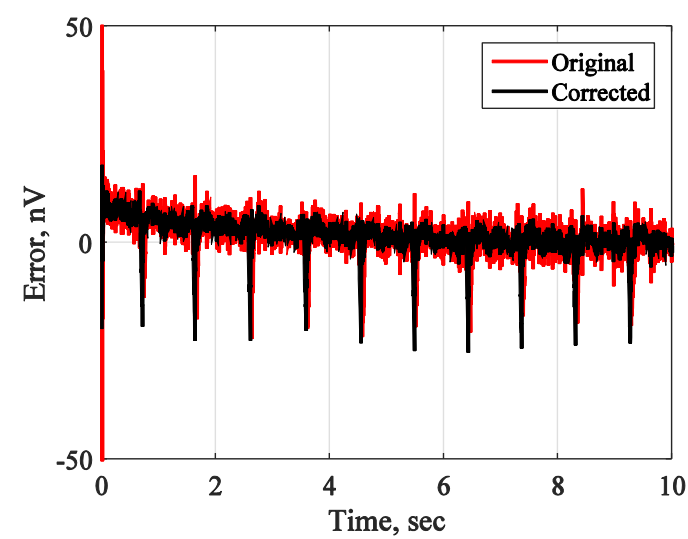

Fig. 13. Amplitude difference between the input and output of the decimation chain, without (original - red) and with compensation (corrected - black).

The average of 6 RMSE values of data records obtained from decimation chain without compensation that were calculated according to (10) is $15 n V$ with a maximum value of $20 n V$ and a minimum of $10 n V$. and for the one with compensation it is $13.2 n V$ with a maximum of $15.5 n V$ and minimum of $0.7 n V$. The most obvious amplitude differences are observed at the QRS peaks where the frequency content of the QRS complex is exposed to a higher group delay. The largest peak amplitude errors in 6 of the data demodulated with decimation filter without and phase correction were $43 n V$ and $44 n V$. According to the normal variation limits given for the ECG wave amplitudes (Table I) 
both mean errors and the maximum QRS peak errors are acceptable since they are relatively small. In addition, the overall mean of input/output dissimilarity calculated by (10) $0.2 \times 10^{-3} \%$ and $0.13 \times 10^{-3} \%$ for uncompensated and compensated filters respectively and waveform dissimilarities for each data records are shown in Fig 14

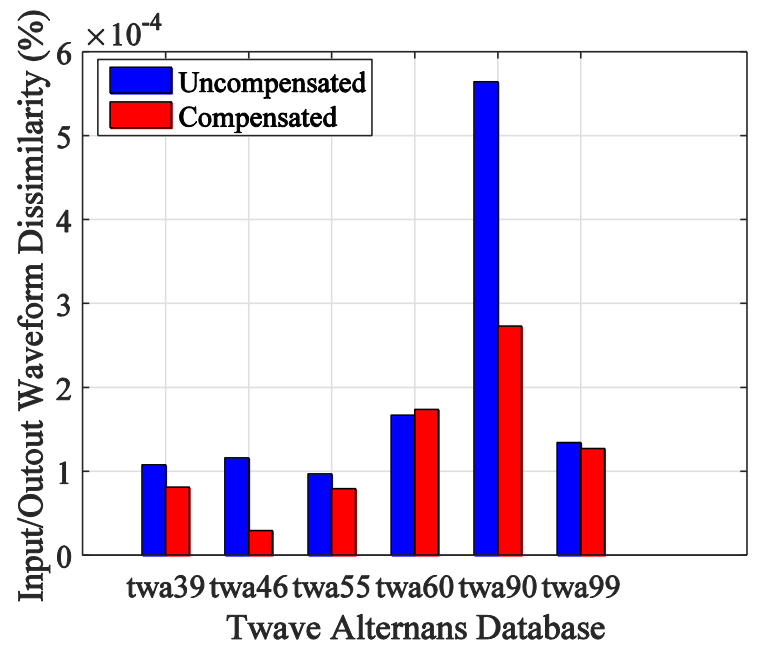

Fig. 14. Waveform Dissimilarity between the input and output of the overall decimation chain without (blue) and with group delay compensation (red).

Finally, the spectral similarity is measured by calculating the distortion ratio as well as the CSC between the input and the output. The DR is calculated for 6 of the ECG data using (11), which are represented in Fig. 15 and the means are $2.2 \%$ and $2.92 \%$, for filters without and with compensation, respectively. The CSC for the input and the output is also calculated which give values that are approximately 1 for most of the frequencies, showing high spectral coherence between the input and output. 


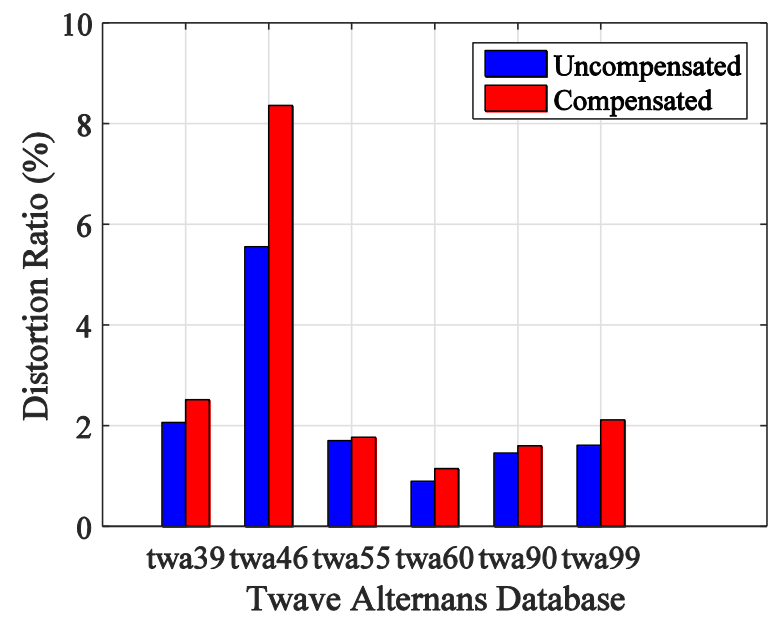

Fig. 15. Input/ Output Distortion Ratios of the overall decimation chain without (blue) and with phase compensation (red).

The above mentioned experiment is repeated for the data obtained from MIT-BIH which are digitized at $360 \mathrm{~Hz}$ and oversampled by a factor of 128 (i.e. $46.08 \mathrm{kHz}$ ). Average of the RMSE values obtained from decimation chain without compensation is $83.5 n V$ and for the one with compensation is $75 \mathrm{~V}$. The largest peak amplitude errors in 13 of the data demodulated with decimation filter without and phase correction are 48 $n V$ and $60 n V$. A 10 second long Lead II recording from a patient suffering from ventricular tachycardia (record 213) after processing with the decimation filters discussed above is presented in Fig. 16. The error measures between the input and output from original and compensated filter are given in Fig 17. 


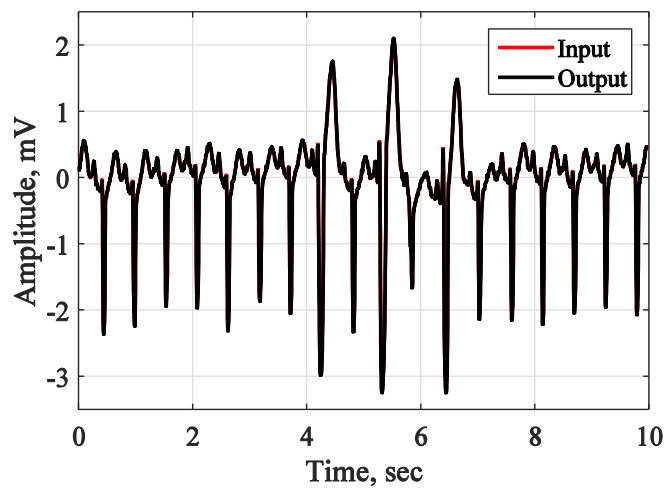

(a)

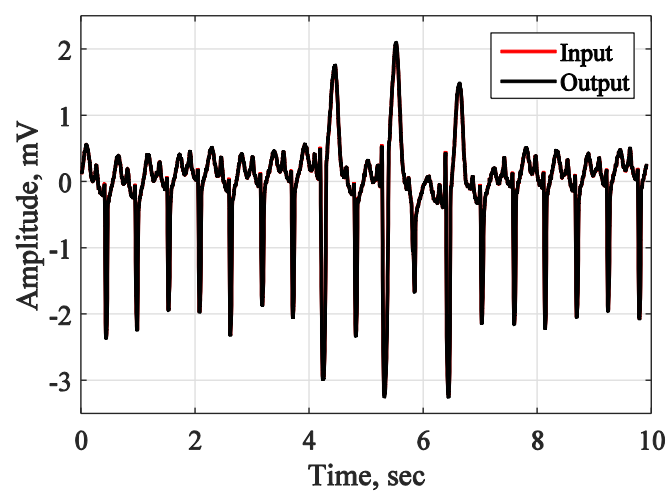

(b)

Fig. 16. Decimation chain output (black) versus the input (red) (a) without phase compensation and (b) with phase compensation (Ventricular Tachycardia)

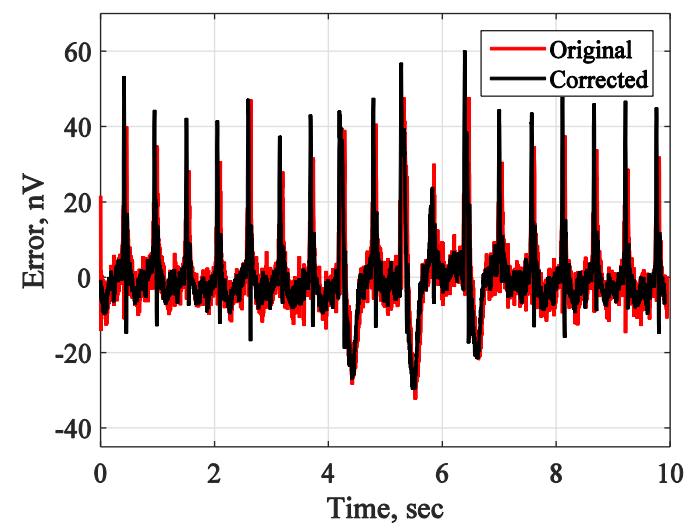

Fig. 17. Amplitude difference between the input and output of the decimation chain, without (red) and with compensation (black). (Ventricular Tachycardia)

Fig. 18 and 19 show the waveform dissimilarity and the measured distortion ratio for these records. The mean DR is calculated to be $2 \%$ and $2.6 \%$ for decimation filter without and with compensation, respectively. Also, the overall mean of input/output dissimilarity is estimated to be $0.13 \times 10^{-3} \%$ and $0.05 \times 10^{-3} \%$ for uncompensated and compensated filters respectively. The group delay compensation results in a better performance by resulting in a lower waveform dissimilarity. 


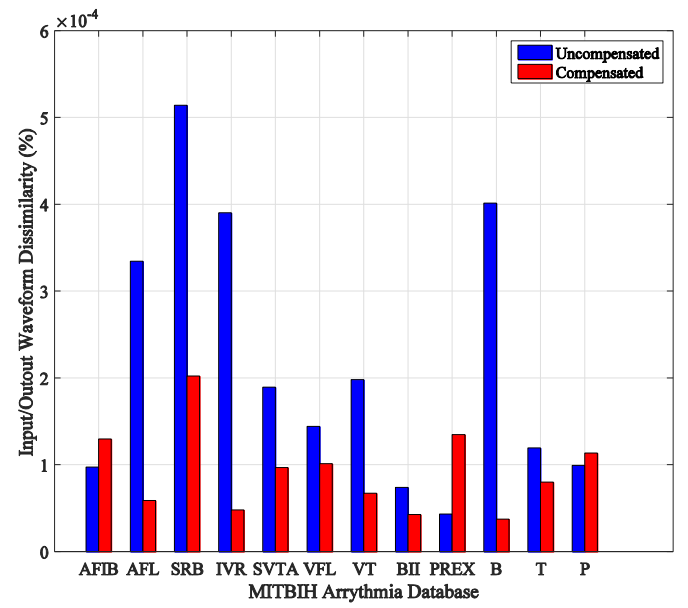

Fig. 18. Waveform Dissimilarity between the input and output of the overall decimation chain without (blue) and with phase compensation (red).

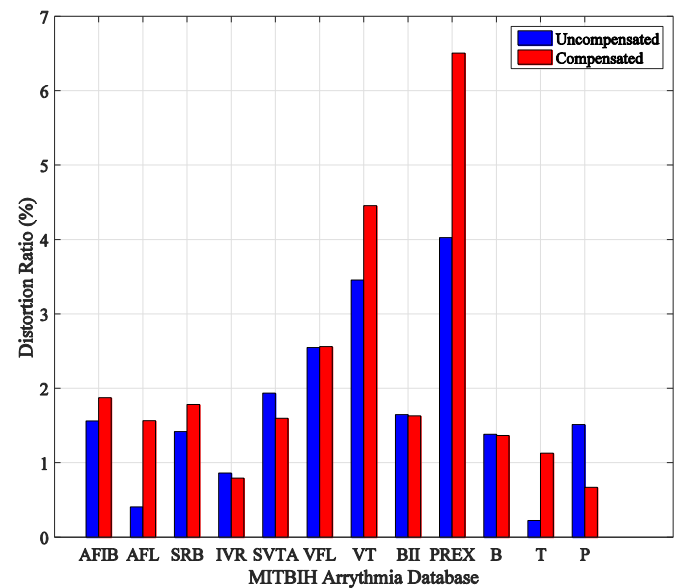

Fig. 19. Input/ Output Distortion Ratios of the overall decimation chain without (blue) and with phase compensation (red).

The performance of the proposed polyphase filter is also compared to the AP based polyphase filters that are designed according to the algorithms provided in the state-ofthe art. Cascading multiples of the polyphase structures improves the stopband attenuation while keeping the cost and complexity low, however in the cost of decreased passband ripples and increased group delay. In order to achieve a better stopband attenuation a cascade of two proposed polyphase filters are implemented [7]. According 
to the algorithm provided in [37], a two path polyphase filter with two cascaded AP filters in both paths and finally, the filter designed in [38] incorporating 4 cascaded two path polyphase structures with single AP filters are implemented. Table III shows the filter specifications, the number of adders and multipliers required and the group delay introduced by each filter.

TABLE III. TWO-PATH AP BASED HB IIR FILTER CHARACTERISTICS CSOMPARISON WITH THE STATE-OF-THE-ART

\begin{tabular}{|c|c|c|c|c|c|c|c|}
\hline & \multicolumn{3}{|c|}{ Filter Specifications } & \multicolumn{2}{|c|}{ Cost } & \multicolumn{2}{|c|}{$\begin{array}{c}\text { Group Delay Variation } \\
\text { (Samples) }\end{array}$} \\
\hline Filter & Order & $\begin{array}{rc}\varepsilon_{p}^{*} & \varepsilon_{s}^{* *} \\
(\mathrm{~dB}) & (\mathrm{dB})\end{array}$ & $\mathbf{v T} * * *$ & $\begin{array}{l}\text { No. of } \\
\text { Adders }\end{array}$ & $\begin{array}{l}\text { No. of } \\
\text { Multipliers }\end{array}$ & $0-0.0625$ & $0-0.125$ \\
\hline Our Work & 5 & $0.47 \mu \quad 70$ & 0.15 & 6 & N/A & 1.86 & 7.61 \\
\hline [7] & 10 & $1 \mu \quad 140$ & 0.15 & 12 & N/A & 3.44 & 14.17 \\
\hline [37] & 9 & $1.17 \mu \quad 70$ & 0.05 & 9 & 4 & 3.01 & 12.45 \\
\hline [38] & 12 & $0.1 \quad 88$ & 0.05 & 11 & N/A & 3.63 & 15.32 \\
\hline
\end{tabular}

\section{Discussion}

The calculated SNR at the modulator and decimation filter output has shown that the proposed design with or without compensation successfully filters the out-of-band quantization noise while preserving the SNR in the signal band without detrimental group delay distortion. According to the simulation results obtained by using various ECG data sets with different diagnostic importance, the spectral similarity for both sets of data (healthy and unhealthy) is approximately the same due to the passband characteristics of the decimation filters. Morphological similarity between the filter input and the output is too high for the filters without compensation in the case of both normal sinus rhythm and arrhythmia. In addition, since the mean amplitude error is 
negligibly small and almost the same for both filters, the use of the compensation filter is not necessary, thus saving power and cost by avoiding the use of extra hardware.

The comparisons with the state-of-the art filters and the filters implemented according to the state-of-the-art algorithms showed that the proposed polyphase structures are superior to others since they provide very small passband ripples and sufficient stopband attenuation with negligible group delay variation in the band of interest. The proposed design requires no multiplier which makes it superior to the filter designed by [37], although they exhibit almost the same magnitude characteristics. Although, [38] is a multiplier free design also, it requires almost twice the number of adders in order to achieve a stopband attenuation of $80 \mathrm{~dB}$ and suffers from having large passband ripples. In addition, the group delay variation introduced by our design is almost half of the group delay variation introduced by [37] and [38]. Cascading two polyphase structure provides a very high stopband attenuation and micro $\mathrm{dB}$ passband ripples. However, the performance of a single polyphase filter incorporated in a decimation chain is sufficient enough to attenuate the out-of-band quantization noise and prevent aliasing, therefore there is no need for using higher order filters with increased group delay variation.

\section{Conclusions}

The phase non-linearity of the IIR polyphase filters, do not cause a significant distortion on the morphological and spectral characteristics of the input ECG signal. This is due to the very narrow and low frequency range corresponding the physiologically significant frequencies for the ECG signals. In other words, these frequency bands are close to DC where the group delay variation is already minimal (minimum phase filter) without using any group delay compensator. The high spectral coherence, high morphological 
correlation and low $\mathrm{nV}$ error between the input and the output signals quantifies that the IIR polyphase filter introduces minimal distortion to the signal which would not affect critical diagnosis therefore, phase compensation is not a must for such an application. The work reported in this paper is that of a decimation filter to be used in an ECG data acquisition systems with very efficient filtering performance that delivers low-power and low-complexity. The proposed design meets the requirements of demodulating a $\Sigma \Delta$ modulator output while preserving the diagnostically important morphological features of the ECG signal.

\section{Acknowledgment}

The authors wish to thank University of Westminster, Faculty of Engineering for $\mathrm{PhD}$ Studentship. 


\section{References}

[1] S. Chang Chien, C. Hsieh, M. P. Lin, Q. Fang and S. Lee, "Implementation of a realtime ECG signal processor," in IEEE International Symposium on Bioelectronics and Bioinformatics (ISBB), April 2014, pp. 1-4.

[2] G. D. Clifford, F. Azuaje and P. McSharry, "ECG statistics, noise, artifacts, and missing data," in Advanced Methods and Tools for ECG Data Analysis, 1st ed., Eds. Norwood, MA, USA: Artech House, Inc., 2006, pp. 55-65.

[3] P. E. McSharry, G. D. Clifford, L. Tarassenko and L. Smith, "A dynamical model for generating synthetic electrocardiogram signals," IEEE Transactions on Bio-Medical Engineering, vol. 50, pp. 289-294, March 2003.

[4] J. J. McKee, N. E. Evans and D. Wallace, "Sigma-delta analogue-to-digital converters for ECG signal acquisition," in Proceedings of the 18th Annual International Conference of the IEEE Engineering in Medicine and Biology Society, Bridging Disciplines for Biomedicine. Nov 1996, pp. 19-20.

[5] P. K. Jain and A. K. Tiwari, "Heart monitoring systems-A review," Comput. Biol. Med., vol. 54, pp. 1-13, Nov 2014.

[6] R. C. Morling, I. Kale, S. Morris and F. Custode, "DSP engine for ultra-low-power audio applications [codec application]," in Proceedings of the 2003 International Symposium on Circuits and Systems, ISCAS'03, May 2003, pp. 357-360.

[7] Y. Eminaga, A. Coskun, S. A. Moschos and I. Kale, "Low complexity all-pass based polyphase decimation filters for ECG monitoring," in 11th Conference on Ph. D. Research in Microelectronics and Electronics (PRIME), Jul 2015, pp. 322-325.

[8] I. Kale, R. C. Morling, A. Krukowski and D. A. Devine, "A high fidelity decimation filter for sigma-delta converters," in Second International Conference on Advanced A-D and D-A Conversion Techniques and their Applications (ADDA '94), Cambridge, UK, 6-8 Jul 1994, pp. 30-35.

[9] I. Kale, A. Krukowski and N. Murphy, "On achieving micro-dB ripple polyphase filters with binary scaled coefficients," in Second International Symposium on DSP for Communication Systems, Signal Processing Research Institute Adelaide (SPRI), South Australia, April 1994, pp. 26-29.

[10] I. Kale, R. C. Morling, A. Krukowski and D. A. Devine, "Architectural design simulation and silicon implementation of a very high fidelity decimation filter for sigma-delta data converters," in Proceedings IEEE Instrumentation and Measurement Technology Conference (IMTC/94), Hamamatsu, Shizuoka, Japan, 10-12 May 1994, pp. 878-881. 
[11] I. Kale and R. C. Morling, "Sigma-delta modulation and polyphase filtering - the key to high-fidelity data conversion and instrumentation," in Proc. IMEKO TC4 Workshop "ADC Modelling”, invited Paper, Slovak Republic, 7-9 May 1996, pp. 196205.

[12] I. Kale and R. C. Morling, "High Resolution Data Conversion via S-D Modulators and Polyphase Filters: A Review"," In Proc. Measurement, Journal of the International Measurement Confederation (IMEKO), Elsevier Science Publisher, vol. 19, pp. 159168, Nov/Dec 1996.

[13] A. Krukowski and I. Kale, "Polyphase IIR Filter Banks for Subband Adaptive Echo Cancellation Applications," Submitted to IEEE Transactions on Circuits and Systems IITransaction Briefs, May 2002.

[14] I. Kale, "An integrated ultra-low-power DSP engine for biomedical applicationsfrom algorithm to custom silicon realization," in Isik 2003 Workshop on New Challenges in Biomedical Engineering, invited Keynote Lecture, Isik University, İstanbul, Turkey, 14-16 July 2003, .

[15] N. Van Helleputte, M. Konijnenburg, J. Pettine, D. Jee, H. Kim, A. Morgado, R. Van Wegberg, T. Torfs, R. Mohan and A. Breeschoten, "A $345 \mu \mathrm{W}$ multi-sensor biomedical SoC with bio-impedance, 3-Channel ECG, motion artifact reduction, and integrated DSP," Solid-State Circuits, IEEE Journal Of, vol. 50, pp. 230-244, 2015.

[16] X. Liu, Y. Zheng, M. W. Phyu, F. Endru, N. V and B. Zhao, "An ultra-low power ECG acquisition and monitoring ASIC system for WBAN applications," Emerging and Selected Topics in Circuits and Systems, IEEE Journal On, vol. 2, pp. 60-70, 2012.

[17] D. McDonagh, O. Eljamaly and A. Burdett, "1V 14uW switched-opamp $\Delta \Sigma$-ADC for bioelectric data aquisition," in 4th IEEE/EMBS International Summer School and Symposium on Medical Devices and Biosensors, ISSS-MDBS. Aug 2007, pp. 147-150.

[18] T. H. Teo, X. Qian, P. Kumar Gopalakrishnan, Y. S. Hwan, K. Haridas, C. Y. Pang, H. Cha and M. Je, "A 700-W Wireless Sensor Node SoC for Continuous RealTime Health Monitoring," IEEE Journal of Solid-State Circuits, vol. 45, pp. 2292-2299, Nov 2010.

[19] K. Muthusamy, T. Hui Teo and Y. P. Xu, "A 1-V 32- $\mu$ W 13-bit CMOS sigmadelta A/D converter for biomedical applications," in IEEE 8th International Conference on ASIC. ASICON'09. Oct 2009, pp. 207-210.

[20] S. Lee, T. Chen and H. Chiueh, "A multi-channel multi-mode physiological signals acquisition and analysis platform," in IEEE International Symposium on Circuits and Systems (ISCAS), Beijing, 19-23 May 2013, pp. 397-400.

[21] S. Y. Lee, J. H. Hong, C. H. Hsieh, M. C. Liang, S. Y. Chang Chien and K. H. Lin, "Low-power wireless ECG acquisition and classification system for body sensor networks," IEEE J. Biomed. Health. Inform., vol. 19, pp. 236-246, Jan, 2015. 
[22] S. Lee and C. Cheng, "A low-voltage and low-power adaptive switched-current sigma-delta ADC for bio-acquisition microsystems," IEEE Transactions on Circuits and Systems I: Regular Papers, vol. 53, pp. 2628-2636, Dec 2006.

[23] J. Garcia, S. Rodriguez and A. Rusu, "A Low-Power CT Incremental 3rd Order Sigma Delta ADC for Biosensor Applications," Circuits and Systems I: Regular Papers, IEEE Transactions On, vol. 60, pp. 25-36, 2013.

[24] S. Tao and A. Rusu, "A power-efficient continuous-time incremental Sigma-Delta ADC for neural recording systems," Circuits and Systems I: Regular Papers, IEEE Transactions On, vol. 62, pp. 1489-1498, 2015.

[25] Y. Yao, K. Zhang, H. Chen and Y. Cheng, "The decimator with multiplier-free realizations for high precision ADC applications," in IEEE 10th International Conference on ASIC (ASICON), Oct 2013, pp. 1-4.

[26] C. Tsou, C. Hsieh, M. Liang, P. Huang and S. Lee, "ECG acquisition system with heart rate detection and energy harvesting for drivers," in Bioelectronics and Bioinformatics (ISBB), 2015 International Symposium On, 2015, pp. 31-34.

[27] R. C. Morling, I. Kale, C. Tsang, S. Morris, G. Hague and C. Foran, "The design of a sigma-delta codec for mobile telephone applications," in Second International Conference on Advanced A-D and D-A Conversion Techniques and their Applications, Jul 1994, .

[28] P. P. Vaidyanathan, "Fundamentals of multirate systems," in Multirate Systems and Filter BanksAnonymous Englewood Cliffs, New Jersey: Prentice-Hall, Professional and Technical Division, 1993, pp. 100-178.

[29] I. Kale, R. C. Morling, A. Krukowski and C. Tsang, "A high-fidelity decimator chip for the measurement of Sigma-Delta modulator performance," IEEE Transactions on Instrumentation and Measurement, vol. 44, pp. 933-939, Oct 1995.

[30] A. Krukowski and I. Kale, DSP System Design: Complexity Reduced IIR Filter Implementation for Practical Applications. Dordrecht: Kluwer Academic Publisher, 2003.

[31] S. Cetinsel, R. C. Morling and I. Kale, "An FPGA based decimation filter processor design for real-time continuous-time $\Sigma-\Delta$ modulator performance measurement and evaluation," in 20th European Conference on Circuit Theory and Design (ECCTD), Aug 2011, pp. 397-400.

[32] G. Moody, "The PhysioNet / Computers in Cardiology Challenge 2008: T-Wave Alternans," Computers in Cardiology, vol. 35, pp. 505-508, 2008.

[33] A. L. Goldberger, L. A. Amaral, L. Glass, J. M. Hausdorff, P. C. Ivanov, R. G. Mark, J. E. Mietus, G. B. Moody, C. K. Peng and H. E. Stanley, "PhysioBank, 
PhysioToolkit, and PhysioNet: components of a new research resource for complex physiologic signals," Circulation, vol. 101, pp. E215-20, Jun 13, 2000.

[34] G. B. Moody and R. G. Mark, "The impact of the MIT-BIH arrhythmia database," IEEE Engineering in Medicine and Biology Magazine, vol. 20, pp. 45-50, May-June 2001.

[35] J. R. Buck, M. M. Daniel and A. C. Singer, Computer Explorations in Signals and Systems using MATLAB. Upper Saddle River, USA: Prentice-Hall, Inc., 1997.

[36] C. M. Hymel, M. H. Skolnick, R. A. Stubbers and M. E. Brandt, "Temporally advanced signal detection: A review of the technology and potential applications," IEEE Circuits and Systems Magazine, vol. 11, pp. 10-25, Aug 2011.

[37] R. Valenzuela and A. Constantinides, "Digital signal processing schemes for efficient interpolation and decimation," Electronic Circuits and Systems, IEE Proceedings $G$, vol. 130, pp. 225-235, 1983.

[38] F. Harris, "Implementing high performance, low computation, IIR filters with 2path recursive all-pass filters and the harris-sharpening filter," in 16th International Conference on Digital Signal Processing, Jul 2009, pp. 1-5. 